\title{
Receptor guanylyl cyclase-G-deficient mice are protected against renal ischemia-reperfusion injury by preventing apoptosis and inflammation
}

\author{
Ruey-Bing Yang*1,2, Heng Lin ${ }^{3}$, Ching-Feng Cheng ${ }^{1,4}$, Hsin-Han Hou ${ }^{1}$, Wei- \\ Shiung Lian ${ }^{1,5}$, Bambang Djoko ${ }^{1}$, Ming-Tzu Tsai ${ }^{1}$ and Chien-Jui Cheng ${ }^{6}$
}

\begin{abstract}
Address: ${ }^{1}$ Institute of Biomedical Sciences, Academia Sinica, Taipei, Taiwan, ${ }^{2}$ Institute of Pharmacology, School of Medicine, National Yang-Ming University, Taipei, Taiwan, ${ }^{3}$ Institute of Pharmacology and Toxicology, School of Medicine, Tzu Chi University, Hualien, Taiwan, ${ }^{4}$ Department of Pediatrics, Tzu Chi General Hospital, Taipei Branch, Taipei, Taiwan, ${ }^{5}$ Department of Animal Science and Technology, National Taiwan University, Taiwan and ${ }^{6}$ Graduate Institute of Medical Sciences and Department of Internal Medicine, School of Medicine, Taipei Medical University, Taipei, Taiwan
\end{abstract}

Email: Ruey-Bing Yang* - rbyang@ibms.sinica.edu.tw

${ }^{*}$ Corresponding author

from 3rd International Conference on cGMP Generators, Effectors and Therapeutic Implications

Dresden, Germany. 15-17 June 2007

Published: 25 July 2007

BMC Pharmacology 2007, 7(SuppI I):SI8 doi:I0.II86/I47I-22I0-7-SI-SI8

This abstract is available from: http://www.biomedcentral.com/I47I-22I0/7/SI/SI8

(c) 2007 Yang et al; licensee BioMed Central Ltd.

\section{Background}

Guanylyl cyclase-G (GC-G) is the last member of the receptor GC family which has so far been identified. However, the specific endogenous ligands, tissue distribution and function of GC-G remain largely unknown. Our studies in mice demonstrated by RT-PCR and immunohistochemistry that GC-G mRNA and protein are expressed in the kidney, specifically within renal tubular epithelial cells. To elucidate the renal functions of GC-G in vivo, we produced a new genetic mouse model with targeted disruption of the GC-G gene $\left(\mathrm{GC}^{-\mathrm{G}^{-}} / \mathrm{)}\right)$. Because increased expression of GC-G mRNA was observed in response to ischemia-reperfusion $(\mathrm{I} / \mathrm{R})$, we studied renal function of GC-G-/- mice and their wild-type (WT) counterparts not only at baseline but also after I/R.

\section{Materials and methods}

WT and GC-G- $/$ - mice were subjected to bilateral renal artery occlusion (45 min) followed by reperfusion $(24 \mathrm{~h})$. Markers for renal dysfunction, histopathology, apoptosis, and inflammation were evaluated.

\section{Results}

Under normal conditions, no apparent renal histological alterations were observed in GC-G-- mice. However, I/Rinduced renal dsyfunction, as evident by the elevation of serum creatinine and urea levels, was significantly attenuated in GC-G-\% mice compared with WT mice. Furthermore, genetic ablation of GC-G prevented tubular disruption, tubular cell apoptosis, and caspase-3 activation, which was accompanied by a marked reduction in neutrophil infiltration number, myeloperoxidase activity, or induction of proinflammatory cytokine interleukin (IL)- 6 and adhesion molecule P-selectin in renal tissues under I/R in GC- $\mathrm{G}^{-}-$ compared to levels seen in WT mice. In addition, gel mobility shift assay demonstrated that nuclear factor (NF)-кBpromoter binding activity is markedly suppressed under renal I/R in GC-G-deficient mice compared to WT controls.

\section{Conclusion}

Together, our study demonstrates for the first time that GCG may play critical apoptotic and inflammatory roles during I/R-induced acute renal failure. This knockout mouse provides an excellent model to further elucidate the signalling pathway and the (patho)physiological functions mediated by GC-G. 